\title{
Características e manejo do Tumor de Wilms: uma revisão narrativa
}

\author{
Management of Wilms'Tumor: a narrative review
}

Tratamiento del Tumor de Wilms: una revisión narrativa

Júlia Maria Moreira Silva ${ }^{1 *}$, Ana Caroline Schoenberger Kipper²,Bárbara Helena dos Santos Neves ${ }^{3}$,Denise Suptitz Borges ${ }^{4}$, Eleonora Lemos Salmazo ${ }^{5}$, Fernanda Pimenta Fernandes ${ }^{6}$, Gabriela Garbuio Vendramini ${ }^{7}$, Sâmely Camoesi Beltellini ${ }^{8}$, Sara Oliveira Reis ${ }^{1}$, Acimar Gonçalves da Cunha Júnior?.

\section{RESUMO}

Objetivo: Descrever e revisar sobre o Tumor de Wilms (TW), com ênfase no tratamento cirúrgico e discutir a quimioterapia pré ou pós-cirúrgica e os exames necessários, conforme as diretrizes mais atuais disponíveis. Revisão bibliográfica: O TW é um tumor embrionário derivado dos metanefros, células precursoras do rim. É o tumor renal mais comum na infância e a terceira malignidade pediátrica mais comum. Seu tratamento é essencialmente cirúrgico, podendo ou não contar com quimioterapia, de acordo com os protocolos Children's Oncology Group (COG) e International Society of Pediatric Oncology (SIOP). Critérios radiográficos e patológicos são empregados para avaliar a viabilidade pré-operatória, indicando candidatos apropriados para cada cirurgia. A nefrectomia radical é o tratamento de escolha nos casos de tumor unilateral. Já em casos de tumores bilaterais, opta-se pela nefrectomia parcial associada a técnica da cirurgia poupadora de néfrons, objetivando preservar a função renal. É consenso na literatura que o rastreio e diagnóstico precoce conduz a um melhor prognóstico e reduz morbimortalidade. Considerações finais: Conclui-se que o estudo do TW se faz extremamente necessário, dada sua importância epidemiológica mundial. $O$ desenvolvimento de protocolos como COG e SIOP são de grande valia para o avanço do tratamento desses tumores.

Palavras-chave: Tumor de Wilms, Nefroblastoma, Cirurgia oncológica.

\begin{abstract}
Objective: Describe and review the Wilm's Tumor (WT), with an emphasis on surgical treatment and discuss pre or post-surgical chemotherapy and the necessary tests, according to the most current guidelines available. Bibliographic review: WT is an embryonic tumor derived from the metanephros, precursor cells of the kidney. It is the most common kidney tumor in childhood and the third most common pediatric malignancy. Its treatment is essentially surgical, with or without chemotherapy, according to the protocols Children's Oncology Group (COG) and International Society of Pediatric Oncology (SIOP). Radiographic and pathological criteria are used to assess preoperative viability, indicating suitable candidates for each surgery. Radical nephrectomy is the treatment of choice in cases of unilateral tumor. In cases of bilateral tumors, partial nephrectomy associated with the nephron-sparing surgery technique is chosen, aiming to preserve renal function. There is a consensus in the literature that screening and early diagnosis leads to a better prognosis and reduces morbidity and mortality. Final considerations: It is concluded that the study of WT is extremely necessary, given its worldwide epidemiological importance. The development of protocols such as COG and SIOP are of great value for advancing the treatment of these tumors.
\end{abstract}

Keywords: Wilms' tumor, Nephroblastoma, Surgical oncology.

${ }^{1}$ Centro Educacional do Planalto Central Apparecido dos Santos (UNICEPLAC), Gama - DF.

*E-mail: juliamoreira.ms@gmail.com

2 Universidade Federal de Mato Grosso (UFMT), Sinop - MT.

${ }^{3}$ Centro Universitário de Várzea Grande (UNIVAG), Várzea Grande - MT.

${ }^{4}$ Universidade de Gurupi (UNIRG), Gurupi - TO.

5 Universidade de Franca (UNIFRAN), Franca - SP.

${ }^{6}$ Faculdade Ciências Médicas de Minas Gerais (FCM-MG), Belo Horizonte - MG.

7 Universidade de Marília (UNIMAR), Marília - SP.

8 Universidade Anhembi Morumbi (UAM), São José dos Campos - SP.

9 Universidade de Brasília (UnB), Brasília - DF. 


\section{RESUMEN}

Objetivo: Describir y revisar el Tumor de Wilms (TW), con énfasis en el tratamiento quirúrgico y discutir la quimioterapia pre o posquirúrgica y las pruebas necesarias, de acuerdo con las guías más actuales disponibles. Revisión bibliográfica: TW es un tumor embrionario derivado de las metanefros, células precursoras del riñón. Es el tumor renal más común en la infancia y la tercera neoplasia maligna pediátrica más común. Su tratamiento es quirúrgico, con o sin quimioterapia, según los protocolos Children's Oncology Group (COG) y International Society of Pediatric Oncology (SIOP). Se utilizan criterios radiográficos y patológicos para evaluar la viabilidad preoperatoria, indicando candidatos adecuados para cada cirugía. La nefrectomía radical es el tratamiento de elección en casos de tumor unilateral. En casos de tumores bilaterales se opta por nefrectomía parcial asociada a técnica de cirugía conservadora de nefronas, con objetivo de preservar la función renal. Existe un consenso en la literatura de que el cribado y el diagnóstico precoz conducen a mejor pronóstico y reducen la morbilidad y mortalidad. Consideraciones finales: Se concluye que el estudio TW es sumamente necesario, dada su importancia epidemiológica mundial. El desarrollo de protocolos como COG y SIOP son de gran valor para avanzar en el tratamiento de estos tumores.

Palabras clave: Tumor de Wilms, Nefroblastoma, Oncología quirúrgica.

\section{INTRODUÇÃO}

O Tumor de Wilms leva o nome de Max Wilms, primeiro cirurgião alemão que o descreveu. É um tumor embrionário derivado dos metanefros, células precursoras do rim, formadas na quarta semana de gestação, e funcionais a partir da oitava semana. É o tumor renal mais comum na infância e a terceira malignidade pediátrica mais comum, precedida da leucemia e tumores no sistema nervoso central. É particularmente observado na faixa etária abaixo de 5 anos, correspondendo a $90 \%$ dos tumores renais. Geralmente afeta apenas um rim, mas tumores sincrônicos bilaterais ou multifocais ocorrem em cerca de $10 \%$ dos casos (MILLAR AJW, et al., 2017; CERNA-ARROYO A, et al., 2020).

O Tumor de Wilms (hereditário ou esporádico) resulta de alteração em um ou mais genes. Mutações germinativas específicas em um dos genes Wilms tumor gene-1 ou WT1, localizadas no braço curto do cromossomo 11 (banda 11p13) estão associadas a essa doença. Algumas síndromes podem ser associadas aos Tumores de Wilms mais agravados, como Síndrome de Beckwith-Wiedemann, Síndrome de Denys-Drash e a síndrome que inclui tumor de Wilms, malformações geniturinárias e retardo mental (WAGR). Outras doenças menos relatadas (1\%) são geralmente casos familiares em que há uma história de tumores em parentes de primeiro ou segundo grau (MÓNICA MQ e YÉSSIKA AGC, 2018; FARIÑAS AM, et al., 2015).

Frequentemente o tumor se apresenta como uma massa abdominal indolor. Outros sintomas associados podem ser hematúria, dor ou hipertensão arterial, em até 20 a 30\% dos casos. Apresentações atípicas aparecem em $10 \%$ dos pacientes e resultam da compressão de órgãos vizinhos ou infiltração vascular. Primordialmente, utilizamos de sociedades internacionais para buscar um tratamento adequado, por exemplo, a Renal Tumor Study Group da International Society of Pediatric Oncology (SIOP-RTSG), bem como os protocolos do National Wilms 'Tumor Study / Children's Oncology Group (COG). Tais entidades usam parâmetros diferentes para definir a conduta de tratamento da doença: estágio, histologia, ensaios clínicos, fatores clínicos e biológicos, como idade do paciente, tamanho e volume do tumor, resposta à quimioterapia (CERNA-ARROYO A, et al., 2020).

O SIOP aplica um subtipo histológico e estratificado, baseado nas alterações histológicas após quimioterapia; os pacientes são divididos em grupos de baixo, intermediário e alto risco com base no grau de necrose tumoral. No sistema COG de categorização histológica antes da quimioterapia, o conteúdo blastemal tem menos significado prognóstico e não é autonômico do estágio do tumor (DOME JS, et al., 2015).

Em uma análise das cirurgias pediátricas, a nefrectomia radical é o tratamento cirúrgico padrão atual para crianças com tumor unilateral. Já nos tumores renais bilaterais, também conhecidos como doença em estágio $\mathrm{V}$, aplica-se a nefrectomia parcial, utilizando-se a técnica da cirurgia poupadora de néfron (NSS), mais recomendada visando preservar a função renal sem comprometer o risco oncológico. Durante a NSS é feito um procedimento rápido que utiliza a solução de azul de metileno, que vai denunciar algum defeito no sistema coletor na pelve renal depois de ocluir o ureter. Se após essa oclusão for visualizada a solução, 
significa que há um defeito nesse sistema, que precisa ser reparado para manutenção da função renal adequada. Posteriormente, a equipe de patologia avaliará a peça cirúrgica, a fim de conferir as margens de segurança histológica (WARMANN SW e FUCHS J, 2019; VUJANIC GM, et al., 2018).

A morbidade mais temida associada à ressecção de uma grande parte do parênquima renal é a insuficiência renal. Portanto, há um benefício teórico em utilizar a técnica NSS, na importância da preservação do parênquima renal. Os critérios radiográficos e patológicos são empregados para avaliar a viabilidade préoperatória, indicando candidatos apropriados para cada cirurgia (NSS, nefrectomia parcial ou total, cirurgia minimamente invasiva) (KIERAN K e DAVIDOFF AM, 2015). A caracterização dos sobreviventes com risco de desenvolver disfunção renal progressiva é importante para orientar as recomendações de vigilância. A identificação precoce de indivíduos com função renal reduzida aumenta a oportunidade de intervenções para preservar a função renal e reduzir a morbidade e mortalidade associadas à insuficiência renal crônica (INTERIANO RB, et al.,2015).

Este trabalho teve como objetivo descrever por meio de uma revisão narrativa sobre o Tumor de Wilms, focando no tratamento cirúrgico segundo seu respectivo estágio e histologia, bem como, a quimioterapia pré ou pós-cirúrgica e os possíveis exames necessários, conforme as diretrizes mais atuais disponíveis.

\section{REVISÃO BIBLIOGRÁFICA}

O Tumor de Wilms (TW) se configura como uma das malignidades mais comuns entre as neoplasias malignas pediátricas. Histologicamente, após 36 semanas gestacionais, os focos celulares embrionários (restos nefrogênicos) persistentes precedem o tumor, estando presentes em 35 a $40 \%$ dos tumores unilaterais e acima de $90 \%$ nos bilaterais. Além desta, outras formas histológicas são encontradas no Tumor de Wilms, como blastemal, epitelial e estromal, as quais são utilizadas para estratificação de risco do paciente.

Os tumores classificados como de baixo risco incluem todas as histologias que, devido ao tratamento quimioterápico pré-operatório, sofrem necrose. Aqueles classificados como de risco intermediário contemplam os tumores do tipo epitelial, estromal, misto, regressivo e anaplasia focal. Já os pacientes de alto risco são aqueles que apresentam o tumor com histologia blastemal e anaplasia difusa (VUJANIC GM, et al., 2018).

Outra classificação histológica existente é descrita pelo sistema COG, que leva em consideração a presença ou não de anaplasia, dividindo os tumores em histologia favorável (sem anaplasia) e histologia desfavorável (anaplasia focal ou difusa) (DOME JS, et al., 2015).

A saber, anaplasia focal é definida como a presença de dois dos seguintes critérios: 1) grandes figuras mitóticas atípicas tripolares e/ou multipolares; 2) aumento nuclear acentuado, com diâmetros nucleares pelo menos três vezes superiores aos das células adjacentes e, 3) núcleos de células tumorais hipercromáticas, apresentando demarcação tumoral nítida intrarrenal sem agitação nuclear em outras áreas. Já a anaplasia difusa caracteriza-se por uma desordem multifocal, não localizada e irreversível ou focal com agitação nuclear (DOME JS, et al., 2015; VUJANIC GM, et al., 2018).

Desta forma, dada a seriedade da patologia, é de suma importância a atenção aos sinais e sintomas, para que seja realizado um diagnóstico precoce, o que aumenta de forma significativa as chances de sucesso no tratamento (MATA MQ e GAMBOA-CHAVES AY, 2018). Um sinal comum, relatado em até $90 \%$ dos casos, é a presença de uma massa abdominal, sendo, geralmente, unilateral e indolor. Contudo, outros achados clínicos podem estar presentes como episódios de infecção urinária, hematúria, hipertensão arterial e dor abdominal (OLIVEIRA PB, et al., 2018).

O diagnóstico do Tumor de Wilms envolve a história clínica e exame físico do paciente e a análise de exames de imagem como a ultrassonografia (USG), a tomografia computadorizada (TC) e a ressonância magnética (RM). Além disso, em muitos casos é necessária análise histológica para comprovação diagnóstica (ILLADE L, et al., 2018). Assim, é válido destacar que o estudo dos exames de imagem tem papel fundamental não só para o diagnóstico presuntivo, mas também para o planejamento pré-operatório e a avaliação metastática. Destaca-se que a USG deve ser o primeiro exame solicitado por não fornecer radiação 
e ser capaz de garantir uma boa análise dos rins e dos órgãos adjacentes, já a TC apresenta papel chave na visualização de linfonodos e se há invasão de órgãos adjacentes, agregando para o estadiamento e para avaliação após o tratamento (RABELLO L, et al., 2019).

Desse modo, exames de imagem são adotados nos protocolos pré-operatórios, pois são capazes de evidenciar dados valiosos. A ecografia com Doppler é importante em todos os casos para esclarecer o número, tamanho e localização dos focos tumorais, podendo até mostrar presença ou ausência de mobilidade. Uma ressonância magnética também pode ser solicitada para contribuir no planejamento terapêutico, visto que facilita a visualização da fronteira do tecido mole entre o tecido renal e o tumor. Ademais, uma tomografia computadorizada pode ser indicada para avaliar a situação vascular, uma vez que proporciona informações relativas ao número e a morfologia dos vasos renais e seus subsegmentos, essencial para uma ressecção tumoral específica (WARMANN SW e FUCHS J, 2019).

Visando uma padronização terapêutica, os dois protocolos principais utilizados são Children's Oncology Group (COG) e International Society of Pediatric Oncology desenvolvido em 2001 e modificado pela Renal Tumor Study Group da International Society of Pediatric Oncology em 2016 (SIOP-RTSG - protocolo UMBRELLA) (HEUVEL-EIBRINK MM, et al., 2016; CAFFERATA C, et al., 2017).

De acordo com o Protocolo COG, é realizada nefrectomia inicial e quimioterapia adjuvante em todos os casos, exceto nos tumores bilaterais sincrônicos, e possibilita diagnóstico histológico precoce através da coleta de material e informações de estadiamento inalterados pela quimioterapia (HEUVEL-EIBRINK MM, et al., 2016; CAFFERATA C, et al., 2017).

O Protocolo SIOP recomenda sempre iniciar com quimioterapia neoadjuvante de 4 a 6 semanas composta por quatro semanas de Vincristina e Actinomicina-D para doenças localizadas e, seis semanas para doença metastática com adição de Doxorrubicina (HEUVEL-EIBRINK MM, et al., 2016; CAFFERATA C, et al., 2017). Esta tem como objetivo proporcionar redução tumoral para facilitar sua ressecção e diminuição do risco de ruptura e recidiva abdominal, com melhora do prognóstico. Caso não haja resposta, é realizada quimioterapia por mais seis semanas, não excedendo o prazo de 12 semanas (tempo máximo de redução tumoral) (MILLAR AJW, et al., 2017; CERNA-ARROYO A, et al., 2020; EHRLICH P, et al., 2017; HEUVEL-EIBRINK MM, et al., 2016; MATA MQ e GAMBOA-CHAVES AY, 2018; KIERAN K e DAVIDOFF AM, 2015; DOME JS, et al., 2015). Quando não há respostas significativas à quimioterapia, é importante esclarecer o motivo. Sabe-se que uma malignidade com predominância histológica estromal não irá responder a terapia de forma tão satisfatória como uma anaplasia difusa (WARMANN SW e FUCHS J, 2019).

Em relação ao tratamento cirúrgico, este difere-se de acordo com as características do tumor. Nos pacientes com Tumor de Wilms Unilateral (TWU) a abordagem cirúrgica padrão é a nefrectomia radical aberta. Como alternativa menos invasiva há a nefrectomia radical por via laparoscópica, com eficácia oncológica equivalente e que, quando comparada à aberta, possui um pós-operatório com menor riscos de complicações e menor tempo de internação (HEUVEL-EIBRINK MM, et al., 2016).

Ademais, esta pode ser realizada pela abordagem transperitoneal ou retroperitoneal. Ambas as vias apresentam margem de ressecção e taxa de complicações semelhantes, porém, a retroperitoneal está associada a um menor tempo operatório, menor perda sanguínea, contenção do vazamento de urina para o retroperitônio, o que evita reabordagem para drenar, prevenção de lesão intestinal e íleo pós-operatório. Contudo, está associada à maior chance de disseminação de célula tumoral na cavidade abdominal devido ao processo de morcelação, que pode romper a bolsa endoscópica (INTERIANO RB, et al., 2015; LIM IIP, et al., 2015; LIU JB, et al., 2015).

Apesar da nefrectomia radical ser considerada o padrão para tratamento do Tumor de Wilms Unilateral, existe a possibilidade de ser realizada a cirurgia poupadora de néfrons a fim de reduzir o risco de insuficiência renal tardia por hiperfiltração. Entretanto, essa técnica é menos utilizada e precisa atender os seguintes critérios para ser realizada: o tumor deve estar limitado a um polo e ocupar menos de $33 \%$ do rim; a função renal do rim envolvido deve estar preservada; não pode haver nenhuma invasão tumoral no sistema coletor ou na veia renal e deve ser possível deixar margens livres entre o tumor, o tecido renal saudável e estruturas adjacentes (INTERIANO RB, et al., 2015). 
Em relação ao tratamento do Tumor de Wilms Bilateral (TWB), classificado em estágio V, a principal dificuldade é ressecar o tumor preservando no mínimo $50 \%$ do parênquima renal, a fim de evitar insuficiência renal a longo prazo. Portanto, o TWB é tratado com quimioterapia neoadjuvante e tentativa de cirurgia poupadora de néfrons (NSS) por via transabdominal, sendo está o padrão ouro (MILLAR AJW, et al., 2017).

É necessário definir se é possível operar ambos os rins simultaneamente ou fazer isso de forma sequencial. Em casos de tumores pequenos e periféricos se utiliza a técnica de ressecção conjunta. Já quando o tumor é muito grande ou está localizado centralmente, opta-se por retirar primeiro o mais complicado, devido ao possível comprometimento do sistema coletor e vasos renais (observado pela coloração azul de metileno na pelve renal ao ocluir o ureter) com a finalidade de ter ciência da quantidade de parênquima renal funcional antes de operar o lado mais fácil (MILLAR AJW, et al., 2017; WARMANN SW e FUCHS J, 2019).

Sendo assim, a NSS é realizada com facilidade quando o tumor é periférico, no entanto, muitos cirurgiões veem a nefrectomia total como única alternativa em tumores localizados centralmente, pois ela deve ser realizada em um centro com a experiência apropriada, devido maior complexidade da técnica cirúrgica, pois corre o risco de afetar as estruturas centralmente localizada (MILLAR AJW, et al., 2017; WARMANN SW e FUCHS J, 2019).

Ademais, a técnica deve ser realizada com extrema cautela para evitar a penetração do tumor na pseudocápsula. Posteriormente, é realizado estudo histopatológico para verificar as margens. Além disso, é necessário retirar os linfonodos renais hilares e peri-aórticos com a finalidade de descartar disseminação linfática e deve-se investigar extensão tumoral nas veias renal e cava inferior (MILLAR AJW, et al., 2017; WARMANN SW e FUCHS J, 2019).

Mediante ao exposto, a nefrectomia parcial é o procedimento de escolha devido a excisão do tumor com uma parte de tecido renal saudável, contudo não se deve violar os princípios oncológicos, deixando uma margem de 0,5 a $1 \mathrm{~cm}$. Durante a cirurgia, o estadiamento histológico, é realizado a fim de averiguar a integridade das margens tumorais, sendo a base para a definição do tratamento quimioterápico pós-operatório pois nele classifica-se o tumor de acordo com a agressividade de acometimento renal do lado mais comprometido. As drogas utilizadas são Dactinomicina e Vincristina durante 27 semanas mínimas. (WARMANN SW e FUCHS J, 2019; VUJANIC GM, et al., 2018).

De acordo com o protocolo SIOP-UMBRELLA, o TW é estadiado da seguinte forma: estadio I: tumor limitado ao rim ou presente na gordura perirrenal circundado por cápsula fibrosa que o contém, pode apresentar crescimento protuberante na pelve renal ou no ureter, mas sem infiltrá-los, os vasos do seio renal não são afetados, mas o tumor pode acometer vasos intrarrenais; estadio II: há tumor viável na gordura perirrenal sem estar envolvido por uma cápsula fibrosa, mas com margens bem definidas, sendo completamente ressecável; o tumor pode infiltrar o seio renal e seus vasos sanguíneos ou linfáticos, a parede da pelve renal ou o ureter, veia cava ou órgãos adjacentes com exceção da glândula adrenal; estadio III: tumor viável com margem de ressecção presente, com presença de acometimento linfonodal; confirmação microscópica de tumor viável em superfície amostral de ruptura pré ou intra-operatória; trombo tumoral nas margens de ressecção do ureter, veia renal ou veia cava inferior; implantes tumorais em qualquer parte do abdome; tumor infiltrado na superfície peritoneal; qualquer tumor que necessite de biópsia pré-operatória é automaticamente reclassificado para estádio III; estadio IV: presença de metástase hematogênicas (pulmões, fígado, osso e cérebro) ou em linfonodos fora da região abdominopélvica; estadio V: tumor bilateral, sendo que cada lado deve ser subestadiado de acordo com os critérios acima (VUJANIC GM, et al., 2018).

O seguimento pós-cirúrgico é feito através de ultrassonografia após duas semanas da cirurgia por um ano, ultrassonografia abdominal mensal, radiografia de tórax de três em três meses, tomografia computadorizada abdominal a cada seis meses e exames laboratoriais associados a cintilografia renal para avaliar a função renal. Estima-se que esse seguimento precise ser feito de dois a cinco anos e varia conforme a histologia tumoral e o tipo de cirurgia realizada (FERNANDEZ CV, et al., 2017). Sendo assim, em casos de Tumor Wilms localizados e metastático a radiografia de tórax e ultrassonografia abdominal são realizadas a cada três meses nos primeiros dois anos, a cada quatro meses no terceiro, semestralmente no quarto e anualmente no quinto, avaliando a chance de interrupção do controle após esse período (BROK L, et al., 2018). 
Nos casos de Tumor de Wilms bilateral e com restos nefrogênicos, esses exames são realizados a cada dois meses nos dois primeiros anos, nos dois anos seguintes, a cada três meses e a partir disso deve ser realizada anualmente até dez anos, também avaliando quando poderá ser interrompido. Desse modo, esses esquemas de vigilância contribuem, não só para o melhor controle do quadro do paciente, mas também para a detecção precoce de episódios de recaídas (BROK L, et al., 2018).

Por ser um tumor altamente radiossensível, alguns casos podem ser beneficiados pela radioterapia pósoperatória, sendo eles: tumores que tiveram redução da dose de irradiação de 15Gy para 12Gy com histologia favorável que receberam quimioterapia com Vincristina, Doxorrubicina e Actinomicina-D devido alta sobrevida livre de recidiva e sobrevida local; tumores com metástases pulmonares sem resposta completa; pacientes que não receberam tal irradiação durante o tratamento de $1^{a}$ linha, independente da histologia; adultos com tumores em estágio II (discutível) e tumores histológicos de risco intermediário ou alto risco com grandes chances de ruptura pré ou intra-operatória ou depósitos peritoneais macroscópicos (HEUVEL-EIBRINK MM, et al., 2016).

Infelizmente, cerca de 15\% dos pacientes apresentam recidiva, em média, dois anos após a nefrectomia, sendo o pulmão o local mais comum. A sobrevida global depois da nova manifestação é variável, mas se aproxima de $50 \%$. Dessa forma, dada a prevalência e a seriedade do Tumor de Wilms entre as crianças é importante estar a par do seu manejo, desde o diagnóstico até o acompanhamento pós-operatório (BROK L, et al., 2018).

\section{CONSIDERAÇÕES FINAIS}

É de suma importância a responsabilidade do cirurgião pediátrico na escolha mais apropriada para o tratamento do Tumor de Wilms, tanto uni como bilateral. Para isso, conforme foi exposto nesse trabalho, é recomendada a utilização de dois protocolos principais, COG e SIOP. Além disso, a escolha entre as várias opções de tratamento cirúrgicas devem levar em consideração diversos fatores, como a possibilidade de deixar margens livres entre as estruturas no caso de uma nefrectomia parcial, a maior preservação possível do parênquima renal, de modo a preservar suas funções, principalmente em casos de tumor bilateral, e prevenir insuficiência renal tardia, destacando os benefícios da cirurgia poupadora de néfrons quando indicada, além do tempo de recuperação, principalmente por tratar-se de pacientes pediátricos, o que indica um benefício das técnicas laparoscópicas quando passíveis de uso.

\section{REFERÊNCIAS}

1. BROK J, et al. Relapse of Wilms' tumour and detection methods: a retrospective analysis of the 2001 Renal Tumour Study Group-International Society of Paediatric Oncology Wilms' tumour protocol database. The Lancet Oncology, 2018; 19(8): 1072-1081.

2. CAFFERATA C, et al. Outcome of Nephroblastoma treatment according to the SIOP-2001 strategy at a single institution in Argentina. Journal of Pediatric Hematology/Oncology, 2017; 39(1): 50-55.

3. CERNA-ARROYO A, et al. Perfil clínico, epidemiológico y terapéutico del tumor de Wilms pediátrico en Costa Rica. Acta Médica Costarricense, 2020; 62(3): 113-118.

4. DOME JS, et al. Advances in Wilms tumor treatment and biology: progress. Journal of Clinical Oncology, 2015; 33(27): 2999-3007.

5. EHRLICH P, et al. Results of the first prospective multi-institutional treatment study in children with bilateral Wilms tumor (AREN0534): a report from the Children's Oncology Group. Annals of Surgery, 2017; 266(3): 470-478.

6. FARIÑAS AM, et al. Características clinicoterapéuticas de niños y adolescentes con neoplasias renales MEDISAN, 2015;19(7): 821.

7. FERNANDEZ CV, et al. Clinical outcome and biological predictors of relapse after nephrectomy only for very low-risk Wilms tumor: a report from Children's Oncology Group AREN0532. Annals of Surgery, 2017; 265(4): 835-40.

8. HEUVEL-EIBRINK MM, et al. Position paper: rationale for the treatment of Wilms tumour in the UMBRELLA SIOPRTSG 2016 protocol. Nature Reviews Urology, 2017; 14(12): 743-752.

9. ILLADE L, et al. Tumor de Wilms: revisión de nuestra experiencia en los últimos 15 años. Anales de Pediatría, 2018; 88(3): 140-149.

10. INTERIANO RB, et al. Renal function in survivors of nonsyndromic Wilms tumor treated with unilateral radical nephrectomy. Cancer, 2015; 121(14): 2449-2456. 
11. KIERAN K, DAVIDOFF AM. Nephron-sparing surgery for bilateral Wilms tumor. Pediatric Surgery International, 2015; 31(2): 229-236.

12. LIM IIP, et al. Experience with retroperitoneal partial nephrectomy in bilateral Wilms tumor. European Journal of Pediatric Surgery: Official Journal of Austrian Association of Pediatric Surgery, 2015; 25(1): 113-7.

13. LIU JB, et al. Laparoscopic radical nephrectomy of Wilms' Tumor and renal cancer in children: preliminary experience from a two-center study in China. Journal of Laparoendoscopic \& Advanced Surgical Techniques, 2015; 25(6): 516521.

14. MATA MQ, GAMBOA-CHAVES AY. Wilms tumor in Costa Rican children. Acta Médica Costarricense, 2018; 60(1): 1520.

15. MILLAR AJW, et al. Management of bilateral Wilms tumours. Pediatric Surgery International, 2017; 33(7): 737-745.

16. MIRABAL FARIÑAS A, et al. Características clinico terapéuticas de niños y adolescentes con neoplasias renales. MediSan, 2015; 19(7): 821-830.

17. OLIVEIRA PB, et al. Tumor de Wilms bilateral sincrônico: avaliação cirúrgica e sobrevida. Revista Brasileira de Cancerologia, 2018; 64(3): 333-339.

18. RABELLO L, et al. Perfil clínico e radiológico de pacientes com tumor de Wilms em um hospital pediátrico do Sul do Brasil. Arquivos Catarinenses de Medicina, 2019; 48(4):88-98.

19. VUJANIC GM, et al. The UMBRELLA SIOP-RTSG 2016 Wilms tumour pathology and molecular biology protocol. Nature Reviews Urology, 2018; 15(11): 693-701.

20. WARMANN SW, FUCHS J. Technical aspects of Nephron-Sparing Surgery (NSS) in children with bilateral centrally located renal tumors. Seminars in Pediatric Surgery, 2019; 28(6): 150865. 